\section{Space trave}

\section{Dual origins of light flashes seen in space} mena that are observed in space and are caused by the interaction of energetic cosmic-ray particles with the human visual system. Using data gathered on board the Mir space station during the Sileye-2 experiment ${ }^{1}$, we show here that there are two separate components of cosmic rays that cause these flashes: one due to heavy nuclei and one due to protons. This indicates that perception by an astronaut's visual apparatus could involve two complementary mechanisms.

Ever since light flashes in space were predicted $^{2}$ before the first space mission and subsequently reported by early Apollo astronauts, attempts to determine their cause have been made both in space and using ground-based particle accelerators ${ }^{3}$. As a result, several explanations have been proposed to account for the phenomenon (see ref. 4 and references therein).

The measured rate of occurrence of light flashes (LF) varies for different missions: on $\mathrm{Mir}^{5}$, it is much lower (Sileye-1, $0.18 \pm 0.02$ LF min $^{-1}$; Sileye-2, $0.13 \pm 0.01 \mathrm{LF} \mathrm{min}^{-1}$ ) than on Apollo ${ }^{6}\left(0.23 \pm 0.1 \mathrm{LF} \mathrm{min}^{-1}\right)$, Skylab $^{7}\left(1.3 \pm 0.1 \mathrm{LF}^{-1} \mathrm{~min}^{-1}\right)$ and ASTP $\left(0.46 \pm 0.05 \mathrm{LF} \mathrm{min}^{-1}\right)$. This effect is probably due chiefly to a reduction in the speed of low-energy particles by Mir's hull material (aluminium more than $3 \mathrm{~mm}$ thick) and the equipment inside the craft.

As light flashes are caused by particles interacting with the human visual apparatus, their occurrence should be proportional to particle rate (the so-called 'latitude effect'). Particle rates outside the region of the South Atlantic Anomaly (SAA) depend on the geomagnetic cut-off, which is a function of the position-dependent geomagnetic field intensity and direction. The cut-off $C$ represents the minimum rigidity for cosmic rays to reach Mir's orbit without being deflected outwards; it is lower at high geomagnetic latitudes (for Mir's orbit, the lowest cut-off is $C=0.6$ gigavolts (GV)) and higher at the geomagnetic equator (where maximum cut-off is $C=16 \mathrm{GV}$ ). The lower the cut-off, the higher the rate of particles coming from outside the Earth's magnetosphere (we use the vertical cut-off at each location of Mir).

Light-flash and particle rates measured inside Mir were divided in 3-GV bins that separated the regions outside $(C \leqslant 18 \mathrm{GV})$ and inside the SAA $(3 \leqslant C \leqslant 15 \mathrm{GV}$, selected for Earth's magnetic field $B<2.5 \times 10^{-5}$ tesla; Fig. 1). The light-flash rate, $R_{\mathrm{LF}}$, is plotted against the particle rate, $P$, for all particles (almost exclusively protons) in Fig. 1a. In Fig. 1b, $R_{\mathrm{LF}}$ is plotted as a function of the rate of relativistic nuclei, $P_{\mathrm{n}}$, with charge $Z \geqslant 6$, obtained by selecting particles ight flashes are unusual visual pheno-

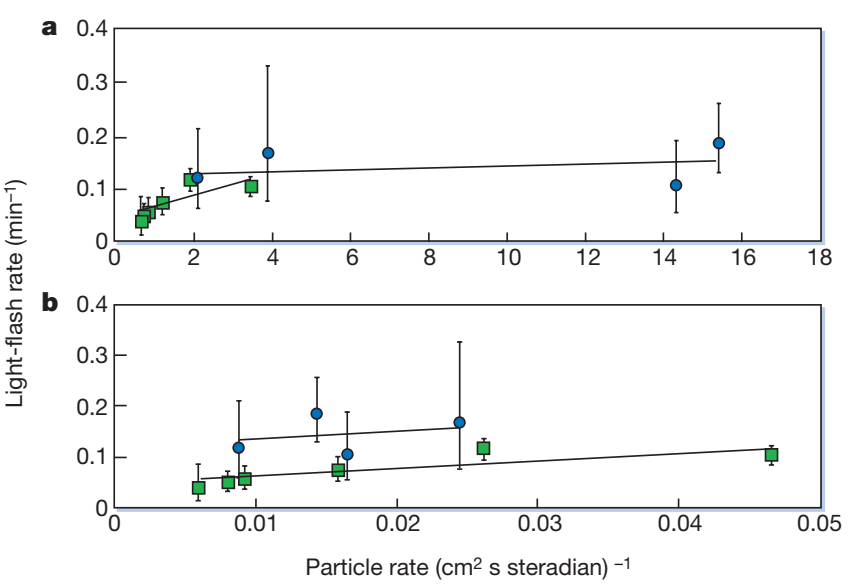

Figure 1 Rate of occurrence of light flashes on board the Mir space station as a function of particle rate for all particles and for relativistic nuclei inside (circles) and outside (squares) the South Atlantic Anomaly. a, Plot of light-flash rate against proton rate; $\mathbf{b}$, light-flash rate

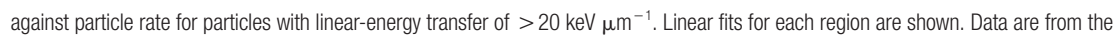
Sileye-2 experiment ${ }^{1}$, in which astronauts wore light-excluding helmets integrated with cosmic-ray particle-flux detectors, enabling the frequency of flashes to be recorded as a function of background flux and orbit position. with high linear-energy transfer $(>20 \mathrm{keV}$ $\mu \mathrm{m}^{-1}$ ) to guarantee the complete removal of the proton component.

From the all-particle plot (Fig. 1a), it is possible to see that $R_{\mathrm{LF}}$ is not linearly proportional to proton flux in all regions: in the SAA, it is roughly independent of proton rate (even though statistical errors preclude any further claim). For instance, at the centre of the SAA $(9 \leqslant C \leqslant 12 \mathrm{GV})$, where particle rate is highest $\left(P=15.4\right.$ particles per $\mathrm{cm}^{2} \mathrm{~s}$ steradian $^{-1}$, where steradian is a unit of spherical angle), the light-flash frequency is $R_{\mathrm{LF}}=0.18$ $\mathrm{min}^{-1}$, which is only slightly higher than in other regions. This implies that the light flashes cannot be caused only by protons in all regions.

Figure $1 \mathrm{~b}$ shows that frequencies of light flashes within the SAA are consistently higher than those for equivalent particle rates outside it, indicating that a further component must contribute to light flashes inside the SAA. Summing all observations in the SAA to reduce statistical errors, we obtain $R_{\mathrm{LF}}=0.15 \pm 0.03 \mathrm{~min}^{-1}$, compared with a rate outside it (in the same cut-off interval of the SAA) of $R_{\mathrm{LF}}=0.06 \pm 0.01 \mathrm{~min}^{-1}$. If heavy nuclei were the only cause of light flashes, these two values should overlap. The existence of two complementary mechanisms for light-flash perception could explain these observations: direct interaction of heavy nuclei with the retina, causing ionization or excitation, and proton-induced nuclear interactions in the eye (with a lower interaction probability) producing knock-on particles.

This explanation is also consistent with previous proposals $s^{3,6-9}$ and the fact that Mir data inside the SAA are halfway between Skylab's (112 light flashes in $12 \mathrm{~min}$ in the SAA) and those of Apollo-Soyuz (no light flashes in the SAA). This is due to the average orbital height and shielding of Mir with respect to the other two space stations. Our results provide further insight into the overall effects of the space environment on humans.

\section{Casolino et al.*}

Department of Physics, University of Tor Vergata, 00133 Rome, Italy

e-mail: casolino@roma2.infn.it

${ }^{*}$ V. Bidoli ${ }^{1}$, A. Morselli ${ }^{1}$, L. Narici ${ }^{1}$, M. P. De Pascale ${ }^{1}$, P. Picozza ${ }^{1}$, E. Reali ${ }^{1}$, R. Sparvoli ${ }^{1}$, G. Mazzenga ${ }^{2}$, M. Ricci' ${ }^{2}$, P. Spillantini ${ }^{3}$, M. Boezio ${ }^{4}$, V. Bonvicini ${ }^{4}$, A. Vacchi ${ }^{4}$, N. Zampa ${ }^{4}$, G. Castellini ${ }^{5}$, W. G. Sannita ${ }^{6}$, P. Carlson ${ }^{7}$, A. Galper ${ }^{8}$, M. Korotkov ${ }^{8}$, A. Popov ${ }^{8}$, N. Vavilov ${ }^{8}$, S. Avdeev', C. Fuglesang ${ }^{10}$ ${ }^{1}$ Department of Physics, University of Tor Vergata, 00133 Rome, Italy; ${ }^{2}$ LNF-INFN, Frascati, Rome, Italy; ${ }^{3}$ Department of Physics, University of Florence, Italy; ${ }^{4}$ Department of Physics, University of Trieste, Italy; ${ }^{5}$ IROE of CNR, Florence, Italy; ${ }^{6}$ NeurophysiopathologyDISMR, University of Genova, Italy, and Department of Psychiatry, State University of New York, Stony Brook, New York, USA; ${ }^{7}$ Royal Institute of Technology, Stockholm, Sweden; ${ }^{8}$ Moscow State Engineering Physics Institute, Moscow, Russia; ${ }^{9}$ Russian Space

Corporation Energia Korolev, Moscow, Russia; ${ }^{10}$ European Astronaut Centre, Cologne, Germany.

1. Bidoli, V. et. al. J. Phys. G 27, 2051-2064 (2001).

2. Tobias, C. A. J. Aviat. Med. 23, 345-372 (1952).

3. McNulty, P. J., Pease, V. P. \& Bond, V. P. Science 201, 341-343 (1978).

4. Horneck, G. Nucl. Track Radiat. Meas. 20, 185-205 (1992).

5. Avdeev, S. et al. Acta Astronautica 50, 511-525 (2002).

6. Pinsky, L. S., Osborne, R. E., Bailey, J. V., Benson, R. E. \& Thompson, L. F. Science 183, 957-959 (1974).

7. Hoffman, R. A., Pinsky, L. A., Osborne, W. Z. \& Bailey, J. Z. NASA Report SP-377, 127-130 (1977).

8. Budinger, T. F. et al. NASA Report SP-412, 193-209 (1977).

9. Charman, W. N., Dennis, J. A., Fazio, G. G. \& Jelley, J. V. Nature 230, 522-524 (1971).

Competing financial interests: declared none. brief communications is intended to provide a forum for both brief, topical reports of general scientific interest and technical discussion of recently published material of particular interest to non-specialist readers. Priority will be given to contributions that have fewer than 500 words, 10 references and only one figure. Detailed guidelines are available on Nature's website (www.nature.com/nature) or on request from nature@nature.com 